\title{
Efecto de épocas de aplicación foliar Bioneat Ag (14-0- 0) en el cultivo de arroz (Oryza Sativa L.)
}

\author{
DOI: https://doi.org/10.33262/ap.v3i4.115
}

\author{
(c) (1) (3) (2)
Effect of foliara application times Bioneat Ag. (14-0-0) in Rice (Oryza Sativa L.)

Eduardo Víctor Jarrin Ruiz. ${ }^{1}$ \& Brenda Narcisa Gurumendi Rodríguez. ${ }^{2}$

\begin{abstract}
This research project was conducted during the dry season of 2014, in the sector Los Lojas, province of Guayas, Ecuador. As general purpose propose new technologies nutrition in rice cultivation to improve the productivity and profitability of the same. As specific objectives: a) Determine the best time of application Bioneat Ag 14 (14-0-0). b) To evaluate the agronomic characteristics of treatments. c) Conduct an economic analysis of treatments. The factors studied were dose applications pursuant Bioneat $\mathrm{Ag} 14$ at different times in the tillering, at panicle initiation, in flowering and combined, tillering and panicle initiation, tillering and flowering, panicle initiation and flowering, tillering and panicle initiation flowering dose of $4 \mathrm{~L} / \mathrm{ha}$. Block design was completely randomized (RCBD) with four replications. Agronomic variables were measured and partial budget analysis was performed in order to measure the economic feasibility of using this product. The highest yield was of $7415.50 \mathrm{~kg} / \mathrm{ha}$ of paddy rice in the application of the $4 \mathrm{~L} / \mathrm{ha}$ in all three phases of crop. The variables studied were no statistically different. Days to flowering, thousand seed weight and yield was achieved having significance statistics. The seven treatment was higher performance.
\end{abstract}

Keywords: Bioneat Ag 14, Foliar applications, Physiology phases, Oryza sativa, Transplant, Performance.

\footnotetext{
${ }^{1}$ Universidad de Guayaquil, Guayaquil Ecuador, eduardo.jarrinr@ug.edu.ec, https://orcid.org/0000-0002$8232-8266$

${ }^{2}$ Universidad de Guayaquil, Guayaquil Ecuador, girlbrenda@hotmail.com, https://orcid.org/0000-00017356-2010
} 


\section{Resumen}

El presente trabajo se desarrolló durante la época seca del 2014 en el sector Los Lojas, Daule provincia del Guayas, Ecuador. Como objetivo general se tuvo que proponer nuevas tecnologías de nutrición en el cultivo de arroz para mejorar la productividad y rentabilidad del mismo. Objetivos específicos: a) Determinar el mejor momento de aplicación de Bioneat Ag 14 (14-0-0). b) Evaluar las características agronómicas de los tratamientos. c) Realizar un análisis económico de los tratamientos. Los factores estudiados fueron aplicaciones de Bioneat Ag. 14, en dosis de 4L/ha, en distintas épocas del cultivo (macollamiento, iniciación de la panícula y floración), para lo cual fueron combinados de la siguiente manera: macollamiento e iniciación de la panícula; macollamiento y floración; iniciación de la panícula y floración; y macollamiento, iniciación de la panícula y floración. Se utilizó un diseño de bloques completamente al azar (DBCA), con cuatro repeticiones. Se midieron variables agronómicas y se realizó el análisis de presupuesto parcial con la finalidad de medir la factibilidad económica del uso de este producto. El mayor rendimiento fue de $7415.5 \mathrm{~kg} / \mathrm{ha}$ arroz paddy con la aplicación de 4L/ha de Bioneat Ag 14 en tres fases del cultivo: macollamiento, iniciación de la panícula y floración. En la mayoría de las variables estudiadas no se tuvo diferencia estadística. En días a floración, peso de mil semillas y rendimiento se logró tener significancia estadística. El tratamiento siete fue el de mayor rendimiento.

Palabras claves: Bioneat Ag. 14, Aplicaciones foliares, Fases fisiologías, Oryza sativa, Trasplante, Rendimiento.

\section{Introducción}

En la actualidad, el cultivo del arroz (Oryza sativa L.) es considerado una gramínea de mucha importancia por ser el alimento básico de millones de personas en todas las regiones del mundo.

En Ecuador, el aumento de la producción, arrocera y sus rendimientos, la reducción de los costos y satisfacción de las demandas de consumo son objetivos comunes de los productores e investigadores en la actualidad. Estas problemáticas hacen que las naciones se preocupen constantemente en mejorar sus conocimientos agrícolas mediante investigaciones y transferencias de tecnologías, para que puedan aumentar su productividad y ser más eficientes en los años venideros (López, 2002; IPNl, 2009).

La mayor área de siembra de arroz en el país está en la costa, con mayor producción en las provincias del Guayas y Los Ríos, con el 58.96\%, 29.81\%, respectivamente; le sigue Manabí con el $6.17 \%$ y las provincias restantes con producciones menores (MAGAP, 2012). 
La superficie cosechada en el 2014 fue aproximadamente de 345.559 ha, con una producción 1518.331 Ton (INEC, 2014), lo que da un rendimiento de 4.39 Ton/ha. (Jarrín-Ruiz. E. 2015).

Hay que destacar, que los últimos años, la producción de arroz se ha mantenido, sin embargo todavía no alcanza el potencial que tienen las variedades mejoradas del país, ya que persisten mal manejo de aplicación de pesticidas, manejo inadecuado de agua y suelo, subutilización de planes nutricionales, etc. (Jarrín- Ruiz. E., 2015). Las limitaciones que afectan al potencial rendimiento del cultivo así, como a su capacidad están relacionados con el desarrollo de nuevas tecnologías existentes. Los agricultores que cultivan esta gramínea lo hacen sin conocer la capacidad de abastecimiento de nutrientes del suelo, los requerimientos nutricionales del cultivo y la asimilación de nutrientes por parte de las plantas. Considerando lo antes expuesto se establecieron los siguientes objetivos:

\section{Objetivo General:}

- Proponer nuevas tecnologías de nutrición en el cultivo de arroz para mejorar la productividad y rentabilidad misma.

\section{Objetivos Específicos:}

- Determinar el mejor momento de aplicación de Bioneat* Ag. Formula 14 (14-0$0)$.

- Evaluar las características agronómicas de los tratamientos.

- Realizar un análisis económico de los tratamientos.

\section{Revisión de literatura}

- Morfología y Fisiología de la planta de arroz

Reyes (2003), señalo que las raíces son delgadas, filerosas, fasciculadas. El tallo es erguido, cilíndrico con nudos, mide de 60 a $120 \mathrm{~cm}$ de altura. Las hojas son alternas. Las flores son de color verde - blanquecina, dispuestas en espiguillas, forman la panícula, colgante a medida que se llena al grano. El fruto es una cariópside.

\section{Fases del Cultivo de arroz}

\section{* Fase vegetativa}

Por lo general dura de 55 a 60 días en las variedades de período intermedio y comprende desde la germinación de la semilla, emergencia y macollamiento (ahijamiento), hasta la diferenciación del primordio floral.

En la fase vegetativa es cuando se determina el rendimiento de una plantación de arroz (Reyes, 2003)

\section{Etapa 0}

De germinación a emergencia: La germinación comienza con la absorción de agua e intercambio gaseoso. Antes del segundo o tercer día después de la siembra, la primera 
hoja sale a través del coleóptilo. El final de la etapa 0 muestra la hoja primaria todavía rizada y la radícula alargada (González y Zamorano, 2009).

\section{Etapa 1}

Plántula: Comprende desde la emergencia hasta inmediatamente antes de aparecer el primer macollo ( $5^{\text {a }}$. Hoja). Su período dura entre 14 y 20 días. Hasta la $3^{a}$ hoja de planta vive de las reservas del endospermo. Se forman raíces secundarias adventicias que serán permanentes (González y Zamorano, 2009).

\section{Etapa 2}

Macollamiento: Ocurra desde la aparición del primer macollo hasta cuando se alcanza el número máximo de ellos. Esta etapa dura de 25 a 55 días. Es difícil distinguir del tallo principal. El crecimiento es muy activo (González y Zamorano, 2009).

\section{Etapa 3}

Elongación del tallo: Comprende desde que el cuarto entrenudo del tallo principal comienza a hacerse notable en longitud, hasta cuando está totalmente elongado (González y Zamorano, 2009).

\section{Fase Reproductiva}

Comprende desde la iniciación de la panícula hasta la floración (Orejuela, 2010).

\section{Etapa 4}

Iniciación de la panícula o primordio: Ocurre desde cuando se inicia el primordio de la panícula hasta cuando la panícula diferenciada es visible como "punto de algodón”. Tiene un lapso de 10 a 11 días (Andrade y Hurtado, 2007).

\section{Etapa 5}

Desarrollo de la panícula: Ocurre desde cuando la panícula es visible como una estructura algodonosa hasta cuando la punta de ella está debajo del cuello de la hoja bandera. Esta etapa demora de 15 a 16 días (Andrade y Hurtado, 2007).

\section{Etapa 6}

Floración: Desde la salida de la panícula de la vaina de la hoja bandera hasta cuando se completa la antesis en toda la panícula. Dura de 1 a 10 días (Ruiz, 2011).

\section{Fase de Maduración}

Comprende desde la floración hasta la madurez del cultivo y dura aproximadamente de 30 a 35 días (Cordero, 1993). 


\section{Etapa 7}

Grano lechoso: Ocurre después de la fertilización de las flores hasta cuando las espiguillas están llenas de un líquido lechoso. Esta etapa comprende de 7 a 10 días (De Datta, 1998).

\section{Etapa 8}

Grano pastoso: Al inicio es acuoso, luego los granos adquieren una consistencia lechosa, luego se torna una masa suave y posteriormente se torna más dura. Su período es de 10 a 13 días (De Datta, 1998).

\section{Etapa 9}

Grano maduro: La panícula comienza a cambiar de color de verde a amarillo; el grano maduro es de color blando y duro y no presenta la tonalidad verde. Esta etapa va desde cuando los granos contienen una consistencia pastosa hasta cuando están totalmente maduros. Su tiempo de duración es de 6 a 7 días (De Datta, 1998).

\section{Fertilización foliar}

La nutrición foliar solamente puede complementar, y en ningún caso sustituir, la fertilización edáfica, debido a que las dosis que pueden aplicarse vía foliar son muy pequeñas. Por esta razón la fertilización foliar es una excelente alternativa para aplicar micronutrientes, los cuales son requeridos en cantidades muy pequeñas por las plantas. (Rodríguez, 2001; Jarrín Ruiz, 2015).

La fertilización foliar es usado en los siguientes casos:

- Para corregir rápidamente deficiencias.

- Frente a problemas de excesiva fijación por parte del suelo.

- Frente a una inadecuada absorción por las raíces (por baja temperatura, daños, etc.)

\section{Etapas de absorción de nutrientes (vía hojas)}

- Mojado de superficie de la hoja con la solución fertilizante. Se usan humectantes para reducir la tensión superficial.

- Penetración a través de infiltración por la cutícula: apoplasto.

- Absorción de nutrientes dentro de la célula (simplasto).

- El movimiento y translocación dependen del movimiento del nutriente en el floema y xilema (Bordoli y Barbazan, 2010).

\section{Pasos para la absorción de nutrientes en las hojas}

- Nutrientes móviles: Los síntomas de deficiencias de los nutrientes móviles se observan en las hojas viejas, ya que la planta los remueve para nuevos puntos de crecimiento. Se distribuyen vía xilema y floema. Entre los nutrientes móviles tenemos los siguientes: N, P, K, Mg y Mo. (Compo Agro, 2004). 
- Nutrientes inmóviles: Los síntomas de deficiencia, de los nutrientes inmóviles se observan en las hojas jóvenes porque estos no pueden ser transportados des las hojas más viejas. Se distribuyen solamente en forma restringida por el floema. Entre los nutrientes inmóviles tenemos los siguientes: S, Zn, Mn, Ca, B, Fe, Cu, (Compo Agro, 2004).

\section{Fertilización con bioneat* ag.}

- Bioneat* Agro (s.f.) Indica que sus productos proporcionan minerales, carbono azucares, nitrógeno, húmicos y excelente sistema de administración, totalmente natural.

- Las plantas, tratadas con estos productos prosperan y florecen de forma adecuada, convencionalmente.

- Las plantas sanas son naturalmente más resistentes a las enfermedades, insectos y hongos.

- El producto formula 14-0-0 de Bioneat* Ag, tiene su contenido total de $14 \%$ de nitrógeno $(\mathrm{N})$, en forma de nitrato.

\section{Materiales y métodos}

El estudio se realizó en época seca del 2014, sector Los Lojas, cantón Daule, provincia del Guayas, con la variedad INIAP-14, en un suelo de textura (\%) arena 40, limo 40 y arcilla 20, según análisis de suelo de los laboratorios INIAP, Litoral Sur, Guayas.

La siembra se realizó de trasplante con plántulas de 21 días edad y a $0.25 \mathrm{~m}$ entre hileras y $0.25 \mathrm{~m}$ entre plantas poniendo 4 a 5 matitas por hoyo. Los datos obtenidos se sometieron a un análisis de varianza y el diseño utilizado experimentalmente fue bloques completamente al azar (DBCA) con ocho tratamientos distribuidos aleatoriamente en cuatro repeticiones en parcelas de $2.5 \mathrm{~m}$ de ancho por $6 \mathrm{~m}$ de largo.

En la comparación de las medias se utilizó la prueba de tu Key al 5\% de probabilidades y los tratamientos estudiados fueron:

\section{Cuadro 1. Tratamientos estudiados - Daule 2015}

\begin{tabular}{cccc}
\hline Tratamientos & $\begin{array}{c}\text { Época de } \\
\text { aplicación }\end{array}$ & Fertilizante Foliar & Dosis (Uha) \\
\hline 1 & M & Bionet $\mathrm{Ag}^{*}$ & 4 \\
2 & I & Bionet $\mathrm{Ag}^{*}$ & 4 \\
3 & $\mathrm{~F}$ & Btonet $\mathrm{Ag}^{*}$ & 4 \\
4 & $\mathrm{M} \mathrm{e} \mathrm{I}$ & Btonet $\mathrm{Ag}^{*}$ & $2+2$ \\
5 & $\mathrm{M} \mathrm{y} \mathrm{F}$ & Bionet $\mathrm{Ag}^{*}$ & $2+2$ \\
6 & $\mathrm{I} \mathrm{y} \mathrm{F}$ & Bionet $\mathrm{Ag}^{*}$ & $2+2$ \\
7 & $\mathrm{M} \mathrm{I} \mathrm{F}$ & Bionet $\mathrm{Ag}^{*}$ & $1.33+1.33+1.33$ \\
8 & Testigo & Testigo & 0 \\
*Bionet Ag: Fórmula 14 (14-0-0); M = Macollamiento; I = iniciación panícula; F = Floración.
\end{tabular}

Fuente: Elaboración propia 
Se realizaron diferentes evaluaciones: Días a cosecha, Altura de planta en $\mathrm{cm}$ ( 5 plantas), Numero de macollas/planta (5 plantas), Numero de panícula/planta (5 plantas), Granos/panícula (5 plantas), Peso de 1000 semillas (g), Rendimiento (kg/ha) y Análisis económico.

Se realizaron diferentes evaluaciones: Días a cosecha, Altura de planta en cm ( 5 plantas), Numero de macollas/planta $\{5$ plantas), Numero de panícula/planta (5 plantas), Granos/panícula (5 plantas), Peso de 1000 semillas (g), Rendimiento (kg/ha) y Análisis económico.

Todos estos muestreos se hicieron en cada una de las réplicas de los diferentes tratamientos y los datos se procesaron por el paquete estadístico Statistical Analysis Sistem "SAS".

\section{Resultados y discusion}

La presente investigación estuvo dirigida a evaluar la eficiencia del Nitrógeno aplicado foliarmente a razón de 4L/ha de Bioneat $\mathrm{Ag}$ : formula 14(14-0-0), en tres fases: macollamiento, iniciación de panícula y floración, con la variedad de arroz Iniap-14

Las mayorías de variable estudiadas no presentaron diferencias estadísticas; en días a floración, peso de 1000 semillas (g) y rendimiento (kg/ha), se logró tener significancia en los tratamientos estudiados.

Cuadro 2: Promedios de dos características agronómicas obtenidas en el experimento "efecto de épocas de aplicación foliar de Bioneat Ag (14-0-0) en el cultivo de arroz (Oryza sativa L.)" Daule, 2015.

\begin{tabular}{ccccc}
\hline Tratamiento & $\begin{array}{c}\text { Época de } \\
\text { aplicación } \\
\text { fisiológica }\end{array}$ & $\begin{array}{c}\text { Dosis de Bioneat Ag } \\
(\mathbf{1 4 - 0 - 0 )} \text { (LVha) }\end{array}$ & $\begin{array}{c}\text { Peso de 1000 } \\
\text { semillas } \mathbf{( g )}\end{array}$ & $\begin{array}{c}\text { Rendimiento } \\
\text { (kg/ha) }\end{array}$ \\
\hline 1 & M & 4 & $19.39 \mathrm{bc}$ & $5039 \mathrm{de}$ \\
2 & $\mathrm{I}$ & 4 & $20.19 \mathrm{ab}$ & $527625 \mathrm{de}$ \\
3 & $\mathrm{~F}$ & 4 & $20.71 \mathrm{a}$ & $5472.25 \mathrm{~cd}$ \\
4 & $\mathrm{M} \mathrm{e} \mathrm{I}$ & $2+2$ & $18.67 \mathrm{~cd}$ & $6010.75 \mathrm{bc}$ \\
5 & M y F & $2+2$ & $20.54 \mathrm{a}$ & $6313 \mathrm{~b}$ \\
6 & I y F & $2+2$ & $18.70 \mathrm{~cd}$ & $6571 \mathrm{~b}$ \\
7 & M I F & $1.33+1.33+1.33$ & $18.24 \mathrm{~d}$ & $7415.50 \mathrm{a}$ \\
8 & Testigo & 0 & $18.07 \mathrm{~d}$ & $4795.50 \mathrm{e}$ \\
& Promedio & & 19.31 & 5861.66 \\
& C.V. (\%) & & 3.31 & 7 \\
\hline
\end{tabular}

Fuente: Elaboración propia

Valores señalados con la misma letra no difieren estadísticamente entre sí (TUKEY al 5\%). Todos los datos se procesaron por el paquete estadístico SAS. Medios con letras en común por columna no difieren significativamente para $\mathrm{p}=0.05$, según la prueba de Tukey. Se puede apreciar que en el cuadro 2 hubo diferencias significativas en el tratamiento 7 con relación al testigo, constituyéndose en el mejor rendimiento con $7415.50 \mathrm{~kg} / \mathrm{ha}$ de arroz paddy, donde se aplicó 4L/ha de Bionetat Ag. Formula 14(14-0- 
0) repartido en las tres fases del cultivo: Macollamiento, Iniciación de Panícula y Floración.

Se logró un incremento de $2620 \mathrm{~kg} / \mathrm{ha}$ de grano de arroz, al comparar el tratamiento siete con el testigo.

Observando el cuadro 2 se puede apreciar que el mejor momento de aplicación del foliar Bioneat Ag 14 es en el macollamiento, inicio de panícula y floración, la dosis de 4L/ha a razón de $1.33 \mathrm{~L}$ en cada fase.

Hay que destacar que el tratamiento siete obtuvo el mayor beneficio neto, con 2522.55 dollares/ha. Este tratamiento resulto no dominado y superior en comparación con las demás combinaciones estudiadas.

\section{Conclusiones:}

- El mayor rendimiento se obtuvo con la aplicación foliar del producto en las tres fases: Macollamiento (M), Iniciación de la Panícula (I) y Floración (F).

- La mayoría de las variables estudiadas no tuvieron diferencias estadísticas. En días a floración peso de 1000 semillas y rendimientos $(\mathrm{kg} / \mathrm{Ha})$ se logró tener significancia en los tratamientos.

- El tratamiento siete fue el de mayor rendimiento, en el mismo que se aplicó el producto en las tres fases del cultivo: macollamiento, iniciación de panícula y floración, en dosis de 4L/ha; el rendimiento del grano fue superior a los $7415,50 \mathrm{~kg} / \mathrm{Ha}$.

- El tratamiento siete resulto no dominado.

\section{Recomendación}

- Realizar aplicaciones en áreas comerciales y otras zonas.

\section{Referencias Bibliográficas}

Andrade, D. y Hurtado, J. 2007. Taxonomía, morfología, crecimiento y desarrollo de la planta de arroz. Manual de cultivo de arroz No. 66 INIAP Boliche Ec. P. 18.

Bioneat Ag (s.f.) Hoja divulgativa del product Bioneat Ag Premium Fertility Foliar Feed Products (en líneas). Disponible en info@bioneat.com - www.bioneat,com p. 1

CIMMYT (Centro Internacional de Mejoramiento de Maíz y Trigo). 1988 Manual metodológico de evaluación económica pp 1-78 México DF.

COMPO Agro, 2004. Compañía fertilizantes foliares y ensayos. Diapositiva \# 4 MX.

González, N. Y Zamorano, D. 2009. El cultivo del arroz (Oryza Sativa L.). Diapositivas $14,15,16$ y 17 Chile. 
INEC (Instituto Nacional de Estadísticas y Censos) 2014 Encuesta de superficie y producción agropecuaria continua ESPAC 2002 - 2011 Ec.

INIAP (Instituto Nacional de Investigaciones Agropecuarias) 2009. Características de la variedad INIAP-14. Ficha técnica de semimor. Disponible en http://www.semimor.com.ec/semillas.htmI p. I.

IPNI (International Plant nutrition Institute) 2009. Fuentes de nutrientes específicas. Disponible en formato PDF en la siguiente dirección: www.iphi.net/specifics

López, M. 2002. Comportamiento orgánico de 16 genotipos promisorios de arroz (Oryza Sativa L.) con cuatro testigos comerciales en la zona de boliche, Provincia del Guayas. Tesis de Ingeniero Agrónomo. Universidad de Guayaquil, Ec. 47p.

Jarrín - Ruiz, E. Comunicación personal, 2015. Guayaquil - Ecuador.

MAGAP (Ministro de Agricultura Ganadería, Acuacultura y Pesca). 2012. Informe situacional de la cadena del arroz. p. 2

Reyes, N. 2003. El cultivo de arroz (Oryza Sativa L.) Manual Técnico para consultores agrícolas y productores programa de arroz. Secretaria de Agricultura y Ganadería. Dirección de Ciencia y Tecnología Agropecuaria. Comayagua, Honduras pp. 910.

Rodríguez. 2001. Memorias curso de fertilidad de suelos. Manejo de suelos y fertilización de arroz. CR. p. 100

Ruiz, N. 2011. Manejo del cultivo de arroz. Informe de pasantías. Programa de Tecnologías en Agricultura p. 17

Surajit K. De Datta. 1998. Producción de arroz: Fundamentos y prácticas. pp. 7-9, 178179.

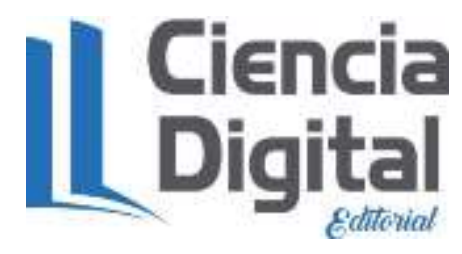




\section{PARA CITAR EL ARTÍCULO INDEXADO.}

Jarrin Ruiz, E. V., \& Gurumendi Rodríguez, B. N. (2021). Efecto de épocas de aplicación foliar Bioneat Ag (14-0-0) en el cultivo de arroz (Oryza Sativa L.). AlfaPublicaciones, 3(4), 86-95. https://doi.org/10.33262/ap.v3i4.115

\section{Ciencia}

El artículo que se publica es de exclusiva responsabilidad de los autores y no necesariamente reflejan el pensamiento de la Revista Alfa Publicaciones.

El artículo queda en propiedad de la revista y, por tanto, su publicación parcial y/o total en otro medio tiene que ser autorizado por el director de la Revista Alfa Publicaciones.
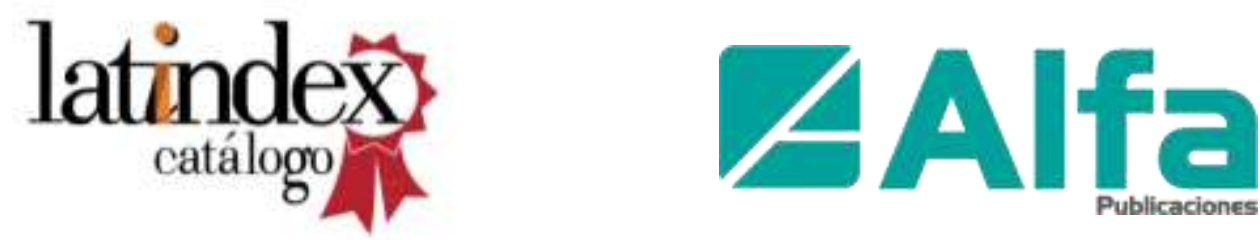